\title{
The interrelationship between metabolic regulation, weight control and obesity
}

By E. A. Newsholme, Department of Biochemistry, University of Oxford, South Parks Road, Oxford $O X_{1} 3 Q U$

Metabolism suffers from the problem that its basic framework, the metabolic pathways, was mainly discovered 40 or 50 years ago, so that it is considered to be a subject in which there is little more to discover, and which is consequently uninteresting. This means that it is usually badly taught and the boredom of learning the details of the pathways usually means that most biologists and clinicians forget the subject as soon as necessary examinations have been taken. Hence the newer developments in metabolic regulation and integration are not always fully appreciated and the fact that such knowledge might be applicable to problems such as weight control, appetite control and obesity are rarely if ever considered. The author discusses, in this paper, the problem of sensitivity in metabolic control, explains the mechanisms for improving sensitivity and suggests that a defect in one of these mechanisms might explain impaired satiety control, impaired thermogenesis and insulin resistance of obese patients. Since this can only be appreciated by a full understanding of mechanisms for improving sensitivity in metabolic control, this subject is discussed in depth before considering weight control and problems of obesity.

\section{SENSITIVITY IN METABOLIC REGULATION}

Sensitivity in metabolic regulation is a measure of the quantitative relationship between the relative change in enzyme activity and the relative change in concentration of the regulator. (If the concentration of a regulator $(x)$ changes by $\Delta x$, the relative change is $\Delta x / x$; similarly if the flux $(J)$ changes by $\Delta J$, the relative change is $\Delta \mathrm{J} / \mathrm{J}$. The sensitivity of $\mathrm{J}$ to the change in $(x)$ is given by the ratio $(\Delta J / J):(\Delta x / x)$ (Newsholme, I 978$)$. For example, if the concentration of a regulator increases two-fold, the question arises, how large an increase in enzyme activity will this produce? The greater the response of enzyme activity to a given increase in regulator concentration, the greater is the sensitivity.

There are several important mechanisms for increasing sensitivity, but before describing these, it is necessary to consider the basic interaction between enzyme and regulator and how this provides a physicochemical limitation to sensitivity in metabolic control. It is important to note that this applies not only to the interaction between enzyme and regulator but also to the interaction between receptor and hormone or receptor and neurotransmitter. 


\section{Equilibrium-binding of a regulator to an enzyme}

It is likely that all regulators modify the activity of an enzyme by binding in a reversible manner to the protein; such binding, which is described as equilibrium-binding, will control the activity of the enzyme as follows:

$$
\underset{\text { inactive }}{\mathrm{E}+\mathrm{R}} \underset{\text { active }}{\rightleftarrows}
$$

where $E$ is in the inactive form of the enzyme and $E^{*}$ is the active form. The asterisk indicates that the binding of $\mathrm{R}$ has changed the conformation of the catalytic site of the enzyme to the active form. The normal response of enzyme activity to the binding of the regulator is hyperbolic. Unfortunately, this response is relatively 'inefficient' for metabolic regulation; for example, a two-fold change in regulator concentration will change the enzyme activity by no more than two-fold (i.e. the maximum sensitivity is unity). This may be difficult to accept when simply observing the steepness of the intial part of a hyperbolic curve. However, it must be appreciated that sensitivity is not the slope of the plot of activity versus concentration of substrate or regulator. Sensitivity is the relationship between the relative change in activity to the relative change in concentration. Since the hyperbolic response is the simplest relationship between protein and regulator it can be considered as the basic response with which any mechanism for improving sensitivity can be compared. Four such mechanisms are described.

\section{Mechanisms for improving sensitivity at non-equilibrium reactions}

Multiplicity of regulators. It has been assumed, in the previous section, that there is only one regulator for the enzyme. However, it is possible for an enzyme to be regulated by several different regulators which bind at different sites on the enzyme. In this case, if the concentrations of all the regulators change in the same direction (or in directions to change the activity of the enzyme in the same way) the effect of all the regulators will be additive.

Co-operativity. For many enzymes that play a key role in metabolic regulation, the response of their activity to the substrate or regulator concentration is sigmoid. This phenomenon is known as positive co-operativity. In this case, the protein is usually polymeric so that it binds more than one molecule of regulator such that the binding of the first molecule of regulator increases the affinity of the other binding sites. Hence the response is no longer hyperbolic and the limitation on sensitivity in regulation is reduced. For part of the concentration range of the regulator, the sensitivity will be greater than that provided by the hyperbolic response, i.e. greater than unity (Newsholme \& Crabtree, I973).

Substrate cycle. A totally different mechanism for improving sensitivity is known as the substrate cycle. It is possible for a reaction that is non-equilibrium in the forward direction of a pathway (i.e. $A \rightarrow B$ ) to be opposed by a reaction that is non-equilibrium in the reverse direction of the pathway (i.e. $B \rightarrow A$ ): 


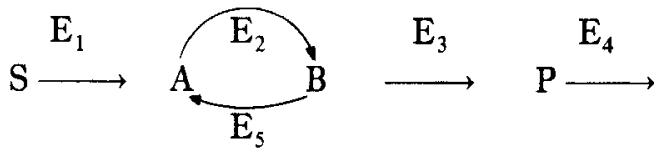

The reactions must be chemically distinct and consequently they will be catalysed by different enzymes (i.e. $\mathrm{E}_{2}$ and $\mathrm{E}_{5}$, above). It is possible that these two opposing reactions are components of two separate pathways which function under different conditions. However, the 'reverse' reaction ( $\mathrm{E}_{5}$ in the above example) may not be part of any pathway and only present in the cell to provide a cycle for metabolic control.

If the two enzymes are simultaneously active, $A$ will be converted to $B$ and the latter will be converted back to $A$, thus constituting the substrate cycle. There are thus two fluxes, a linear flux converting $S$ to $P$ and a cyclical flux between $A$ and $B$. Both fluxes are to a large extent independent and calculations show that the improvement in sensitivity is greatest when the cyclical flux is high but the linear flux is low (i.e. the ratio, cycling rate:flux, is high; Newsholme \& Crabtree, 1976; Newsholme, 1978).

The role of a cycle can best be understood when it is appreciated that, in some conditions, an enzyme activity may have to be reduced to values closely approaching zero. Even with a sigmoid response this would require that the concentration of an activator be reduced to almost zero or that of an inhibitor to an almost infinite level. Such enormous changes in concentration probably never occur in living organisms, since they would cause osmotic and ionic problems and unwanted side reactions. However, the net flux through a reaction can be reduced to very low values (approaching zero) via a substrate cycle. Thus as the product of the forward enzyme $\left(E_{2}\right)$ is produced (i.e. $B$ in the above example) it is converted back to substrate $A$ by the reverse enzyme $\left(E_{5}\right)$. This ensures that the net flux (i.e. $A$ to $B$ ) is very low despite a finite activity of the forward enzyme and a moderate concentration of an activator. Now if the concentration of this activator is increased, by only a small amount above that at which the activities of the two enzymes are almost identical (and the flux is almost zero), the activity of $E_{2}$ will increase so that the net flux through the reaction will increase from almost zero to a moderate rate. Such a cycle therefore provides a large improvement in sensitivity; indeed, it can be seen as a means of producing a threshold (or almost threshold) response with a simple metabolic system.

Since in the substrate cycle both reactions are non-equilibrium, it is not possible to operate even one turn of the cycle without conversion of chemical energy into heat. Usually this comes about by the hydrolysis of ATP to ADP and phosphate since ATP is involved as a substrate in one reaction. Hence the net result of the cycle is the hydrolysis of ATP. For a considerable number of years it was considered that this loss of energy was too high a price to pay and that metabolic control would ensure that such apparently energetically-wasteful cycles would not occur. Indeed, such cycles are sometimes known as 'futile' cycles but there is now considerable evidence to show that these cycles do exist and that the remarkable 
improvement in sensitivity provided by the cycles justifies the metabolic cost to the organism (see Newsholme, 1976, for an historical account of the development of these ideas). Indeed, substrate cycles may operate not only to regulate flux through metabolic pathways but to achieve the controlled conversion of chemical energy (i.e. ATP) into heat, either to maintain body temperature, to raise the temperature (pyrexia) or to reduce body mass by burning off fuel, that is, weight control.

Interconversion cycles. A number of enzymes (e.g. glycogen phosphorylase, pyruvate dehydrogenase) are known to be regulated by a different mechanism from that described above. These enzymes exist in two forms, conventionally designated $a$ and $b$, one being a covalent modification of the other. The conversion of one form to the other is generally brought about by reaction with ATP and in most cases one form is a phosphorylated modification of the other. In general, only one of the two forms $(a)$, has significant catalytic activity so that the flux can be regulated by altering the amount of enzyme in this form. The interconversions between the forms are carried out by enzymes, one for each direction, that catalyse non-equilibrium reactions. The activity of one or both of these enzymes can be altered by regulators. The mechanism of regulation via the conversion of an inactive form of an enzyme into an active form, or vice versa, has similarities to a substrate cycle and, indeed, this 'interconversion cycle' represents a logical extension of the substrate cycle. However, although the improvement in sensitivity provided by such cycles is likely to be large, the precise improvement is difficult to calculate (Newsholme \& Crabtree, I973, I976).

It is important to point out that these four mechanisms are not mutually exclusive. Indeed, it is probable that, at some reactions, all four mechanisms play a role in regulation of flux and this combination could provide an enormous increase in sensitivity.

\section{Sensitivity of near-equilibrium reactions}

Each of the four mechanisms outlined above for improving sensitivity involves some increase in complexity of enzyme mechanism beyond that which gives rise to the basic hyperbolic kinetics. However, regulation of 'near-equilibrium' enzymes by changes in concentration of pathway substrates or cosubstrates is achieved without additional complexity. The improvement in sensitivity depends upon the fact that the rates of the reaction in the forward and reverse directions are considerably greater than the flux (Crabtree \& Newsholme, 1978). The sensitivity depends upon the fact that the catalytic activity is large, a consequence of the high concentrations of such enzymes. This explains why the cell synthesises considerably more enzyme-protein than is required to accommodate the maximum flux through the reaction. The reason that most pathways do not consist of all near-equilibrium reactions is that non-equilibrium reactions provided directionality in a pathway and, furthermore, the sensitivity of the near-equilibrium reactions can only be achieved if they are preceded and followed in the pathway by non-equilibrium reactions (Crabtree \& Newsholme, 1978). 
There is increasing evidence that obesity shortens the lifespan with increased incidence of atherosclerosis, hypertension, gall bladder disease, noninsulin-dependent diabetes mellitus and psychological disturbances. This, together with the large number of individuals in Western societies who suffer from this condition and the changing attitudes of society to obesity, have led to a renewed interest in the causes of the problem. In simple terms, obesity is caused by the ingestion of too much energy and utilization of too little. This is a consequence of the first law of thermodynamics and the existence in the body of metabolic mechanisms for energy storage; energy consumed in excess of requirements will be retained as chemical energy and in the non-growing adult the only major storage form for this energy is triacylglycerol in the adipose tissue. However, there is evidence that a mechanism exists for the maintenance of the normal proportion of adipose tissue ( $120 \mathrm{~g} / \mathrm{kg}$ for men, $260 \mathrm{~g} / \mathrm{kg}$ for women) so that obesity could be caused by an impairment of this mechanism. The fascinating and controversial question is, what is the metabolic basis of this mechanism, knowledge of which might explain the aetiology of obesity? The regulation of body-weight probably involves both control of energy intake and control of energy expenditure and it is suggested that a common metabolic mechanism of control may play an important role in both these processes.

\section{Control of energy intake}

Evidence that an animal can control its food intake is obtained from studies on the effects of stimulation or damage to specific areas of the hypothalamus. Lesions in the lateral hypothalamus lead to anorexia (which can be sufficiently serious that the animal starves to death) whereas stimulation leads to feeding. This led to the idea of a hunger or feeding centre in this part of the brain. On the other hand, lesions in the ventromedial hypothalamus lead to hyperphagia (over-eating) and obesity (if sufficient food is available) whereas stimulation leads to cessation of feeding, thus leading to the idea of a satiety centre. A balance between the two centres would therefore result in normal feeding behaviour. Both glucose and insulin (which will rise in concentration after a meal) probably stimulate the satiety centre and this stimulation depends upon the utilization of glucose by cells in this part of the hypothalamus. In addition, information is received, via nerves, from the mouth and facial area concerning odour, taste, texture and appearance of food; gastric distension may also initiate neural signals to the hypothalamus and the hormones released from the duodenum in response to the entry of food, including cholecystokinin and bombesin, probably provide further sensory input to the centres. How glucose metabolism stimulates the satiety centre is not known, but the author suggests that the problem of sensitivity of this process to small changes in glucose and insulin concentrations arises in these cells as in every other cell. Thus Owen et al. (1979) demonstrate that even after 'feasting', the increase in the blood insulin concentration is between two- and six-fold whereas that of glucose is considerably less than two-fold. Yet such changes must sufficiently 
stimulate the satiety centre that feeding ceases. The author speculates that a substrate cycle, probably between glucose and glucose 6-phosphate in the cells of the satiety centre, could increase enormously the sensitivity of glycolysis to small changes in glucose concentration so that a satisfactory control mechanism is produced (see Surholt \& Newsholme, 1981). Furthermore, if the capacity of this cycle was decreased or if the control mechanism that regulates the activity of this cycle were impaired, the glycolytic system could be insensitive to normal changes in the blood concentration of insulin and glucose so that satisfactory satiety after ingestion of sufficient food may not be achieved.

\section{Control of energy expenditure}

There is considerable evidence that body-weight can be maintained despite marked variations in the food intake. Perhaps the first recorded investigation was carried out by Neuman ( 1902 ) who performed a $725 \mathrm{~d}$ experiment on himself, in which he varied his intake of energy over a wide range and despite this he maintained a constant weight. Similar and more recent studies have been carried out by Gulick (1922) and Passmore et al. (1955). Normal subjects fattened by over-eating require more energy in relation to their body surface for maintenance of the obese state than they require at their natural weight and, also, more than spontaneously obese individuals (Sims et al. 1973). This suggests that these subjects have a natural tendency to 'burn-off' excess food rather than store it. Similarly, feeding rats a high energy diet, in which their normal laboratory diet was supplemented with food palatable to man (so-called 'cafeteria-feeding'), causes a marked increase in heat production after feeding (Rothwell \& Stock, 1979). Experiments in non-ruminant animals and in man have shown that the efficiency of utilization of carbohydrate is considerably higher when the food intake is below maintenance requirements than when the intake is above this requirement (Blaxter, 1970). These observations, together with the first law of thermodynamics, suggest that one or more metabolic processes exist in which excess energy is dissipated in the form of heat.

The question therefore arises as to the nature of the processes that can convert excess chemical energy into heat. One mechanism could be an increase in the activity of substrate cycles.

Substrate cycles and weight control. The role of substrate cycles in improving sensitivity in metabolic control of flux through pathways is discussed above. The improvement is achieved by an increase in the rate of cycling (in relation to the flux through the pathway) which involves an increase in the rate of hydrolysis of ATP to ADP and phosphate. Since cycling does not produce a net metabolic change, except for the hydrolysis of ATP, it results in the conversion of chemical energy into heat. The amount of heat produced will depend upon two factors, the rate of cycling and the number of ATP molecules hydrolysed per turn of the cycle. For the fructose 6-phosphate-fructose bisphosphate cycle one molecule of ATP is hydrolysed for each turn and on the basis of the maximum activity of fructose-bisphosphatase in the muscle of man $\left(2.0 \mu \mathrm{mol} / \mathrm{min}\right.$ per $\mathrm{g}$ at $\left.37^{\circ}\right)$ it can 
be calculated that, if this cycle were fully active in all muscles for $24 \mathrm{~h}$, almost $7000 \mathrm{~kJ}$ of energy could be liberated as heat (Newsholme, 1978). This is more than half of the average daily energy intake. Although it is very unlikely that this cycle would ever be maximally active for a prolonged period, this calculation demonstrates the large capacity for heat production. Indeed, there are a large number of potential substrate cycles in metabolism: glucose-glucose 6-phosphate; glycogen-glucose I-phosphate; fructose 6-phosphate-fructose bisphosphate; phosphoenolpyruvate-pyruvate; triglyceride-fatty acid; fatty acid-fatty acyl-CoA; glycerol-glycerol 3-phosphate; protein-amino acid; RNA-ribonucleotides, cholesterol-cholesterol ester; and this list can also include translocation cycles in which ions (e.g. sodium ions) leak into a cell and are extruded by a pump (i.e. an ATP-requiring reaction) (see Crabtree \& Newsholme, I 978). Consequently, a small stimulation of a large number of such cycles could result in a considerable rate of conversion of chemical energy into heat.

The rate of these cycles might be increased in vivo in at least three different conditions; stress, after exercise and after feeding. It is likely that both anxiety-stress and aggression-stress increase the activity of many cycles involved in the mobilization and utilization of fuels, especially glucose and fatty acids. For example, adrenaline and glucagon increase the rate of the triacylglycerol-fatty acid cycle in adipose tissue (Brooks, 1982). It is well established that in the post-exercise period, oxygen is consumed in excess of that required to support resting metabolism; this is known as the oxygen debt (Hill et al. 1925). It is possible to divide the debt into three phases, the rapid phase, the slow phase and the ultra-slow phase (see Newsholme, 1978). The rapid phase may be explained by replenishment of phosphocreatine stores and re-oxygenation of myoglobin and haemoglobin; the slow phase may be partially explained by reconversion of lactate to glucose and glycogen, but some of the oxygen consumed in this phase (probably as much as $50 \%$ ) cannot be explained by carbohydrate synthesis (see Newsholme, 1978). It is proposed that some of the oxygen consumed in the slow phase and all of the oxygen consumed in the ultra-slow phase is due to the stimulation of substrate cycles. Since the small increase in oxygen consumption associated with the ultra-slow phase may persist for several hours after exercise, it could make a significant contribution to energy expenditure.

The increase in heat production after a meal is known as the thermic response (previously known as the specific dynamic action). Although some of this heat arises from the increased rate of biosynthetic processes, not all can be accounted for in this way. Many of the properties of the thermic response are consistent with a stimulation of cycling rates after a meal (Crabtree \& Newsholme, 1978) and this could be caused by the increased sympathetic drive after feeding (Young \& Landsberg, 1977).

In order for any mechanism to control the amount of triacylglycerol in the adipose tissue, information must be conveyed from the stores to those systems controlling food intake and energy dissipation. Candidates for the role of this messenger include insulin and triiodothyronine. Increases in the concentrations of 
both of these hormones could increase the rate of substrate cycling or the sensitivity to noradrenaline or both. Obesity could result from a low capacity of substrate cycles in different tissues, a reduced ability of the catecholamines to increase the rates of substrate cycling or an impaired mechanism of integration between the catecholamines and insulin (or triiodothyronine) so that, for example, a high blood concentration of insulin would not produce a high rate of cycling in response to catecholamines. Indeed there is evidence of a diminished response to catecholamines in obese human subjects (Jung et al. 1979).

\section{Insulin resistance in obesity}

Insulin resistance is a state in which higher-than-normal concentrations of the hormone are required to produce a given biological effect. This could be caused by the presence of antibodies to the hormone, the production of an abnormal, less effective, insulin or a decreased response of the target tissue to the hormones. In obesity the latter problem is present. Studies in man and experimental animals demonstrate that in obesity the stimulation of glucose utilization in muscle and adipose tissue, the inhibition of lipolysis in adipose, and the inhibition of glucose release by liver are all resistant to insulin. This resistance results in much higher concentrations of insulin being required to maintain normal metabolic functions so that patients are hyperinsulinaemic. In mild hyperinsulinaemia, the number of insulin receptors are reduced, so that in order to obtain the same biological response from a tissue, the concentration of the hormone must be increased. In more severe cases of hyperinsulinaemia, there is not only a decreased number of receptors but also a further post-receptor defect. Thus a very high concentration of insulin (sufficient to saturate all the receptors) does not elicit the maximal biological response observed in the non-resistant state (for reviews see Kahn, I 978; Olefsky \& Kolterman, I98I). The nature of the post-receptor defect is not known. It could be that the sensitivty of the control mechanisms that regulate the key enzymes in tissues is reduced so that a given concentration of the hormone would be less effective, in other words, a decreased rate of substrate cycling.

The author suggest that all three facets of obesity, a reduced satiety response (in respect of the hyperglycaemia and hyperinsulinaemia), a reduced rate of thermogenesis and insulin resistance could be explained by the same defect-a low capacity, or more likely, impaired control of substrate cycles so that the sensitivities of all the basic regulatory processes are reduced.

\section{REFERENCES}

Blaxter, K. L. (1 970). Fedn Proc. Fedn Am. Socs exp. Biol. 30, 1436.

Brooks, B. (1982). PhD thesis, Oxford University.

Crabtree, B. \& Newsholme, E. A. (1978). Eur. F. Biochem. 89, iq.

Gulick, A. (1922). Am. 7 . Physiol, 60, 37 r.

Hill, A. V., Long, C. N. H. \& Lupton, H. (1925). Proc. R. Soc. 97, 96.

Jung, R. T., Shetty, P. S., James, W. P. T., Barrand, M. A. \& Callinghay, B. A. (I979). Nature, Lond. $279,322$.

Kahn, C. R. (1978). Metabolism 27, 1893.

Neumann, R. O. (1902). Arch Hyg. 45, I. 
Newsholme, E. A. (1976). Biochem. Soc. Trans. 4, 978.

Newsholme, E. A. (1978). Biochem. Soc. Symp. 43, 183 .

Newsholme, E. A. \& Crabtree, B. (1973). Symp. Soc. exp. Biol. 27, 429.

Newsholme, E. A. \& Crabtree, B. (1976). Biochem. Soc. Symp. 4r, 6r.

Olefsky, J. H. \& Kolterman, O. G. (198I). Am. F. Med. 70, 5 I.

Owen, O. E., Reichard, G. A. \& Patel, M. S. (1979). Adv. exp. Med. Biol. ri r, irg.

Passmore, R., Meiklejohn, A. P., Dewar, A. D. \& Thow, R. K. (1955). Br. F. Nutr. 9, 27.

Rothwell, N. J. \& Stock, M. J. (1979). Nature, Lond. 281, 3 I.

Sims, E. A. H., Danforth, E., Horton, E. S., Bray, G. A., Glennon, J. A. \& Salans, L. B. (1973). Prog. Horm. Res. 29, 457 .

Surholt, B. \& Newsholme, E. A. (198I). Biochem. J. 198, 62 r.

Young, J. B. \& Landsberg, L. (1977). Nature, Lond. 269, 6 I 5. 